\title{
Psicólogos europeos en los países andinos (Bolivia, Ecuador y Perú) durante la primera mitad del siglo $\mathrm{XX}^{*}$
}

European Psychologists in Andean Countries (Bolivia, Ecuador, and Peru) in the First Half of the 20th Century

Recibido: diciembre 9 de 2013 | Revisado: enero 20 de 2014 | Aceptado: julio 28 de 2014

\author{
RAMÓN LEÓN ** \\ Universidad Ricardo Palma, Lima, Perú
}

doi:10.11144/Javeriana.upsy13-5.pepa

Para citar este artículo: León, R. (2014). Psicólogos europeos en los países andinos (Bolivia, Ecuador y Perú) durante la primera mitad del siglo XX. Universitas Psychologica, 13(5), 1869-1880. http://dx.doi. org/10.11144/Javeriana.upsy13-5.pepa

* El presente trabajo se basa parcialmente en investigaciones llevadas a cabo en el Institut für Geschichte der Psychologie [Instituto de Historia de la Psicología] de la Universidad de Passau (Alemania Federal), durante enero y marzo del 2008, gracias al apoyo financiero de la Konrad-Adenauer-Stiftung [Fundación Konrad Adenauer].

** Correo electrónico: rld310850@yahoo.com.mx

\section{RES U MEN}

Un reducido número de psicólogos y pedagogos europeos trabajó en Bolivia, Ecuador y Perú, los así llamados países andinos, en la primera mitad del siglo XX. En Bolivia, Georges Rouma, un educador belga, fue el responsable de la reforma educativa emprendida por el Gobierno boliviano, organizando una expedición antropológica en los Andes. En Ecuador, Oliver Brachfeld, un psicólogo húngaro de orientación adleriana, se desempeñó por un corto tiempo en la Universidad de Quito, en tanto que en el Perú, Walter Blumenfeld, un psicólogo alemán, obligado a dejar su país en los años del nacionalsocialismo, trabajó en la Universidad de San Marcos desde 1935 y fue un pionero de la orientación objetiva en la psicología en ese país.

Palabras clave

psicólogos europeos; países andinos; siglo XX

\section{A B S T R A C T}

A small number of European psychologists and educators worked in the so called Andean countries, Bolivia, Ecuador and Peru, in the first half of $20^{\text {th }}$. century. In Bolivia Georges Rouma, a Belgian teacher, was responsible for the education reform in the 1910s, and organized an anthropological expedition in the highlands. Oliver Brachfeld, an Hungarian adlerian psychologist, was in Colombia (1965-1966) and Ecuador (1966-1967), where he dead in 1967, teaching at the University of Quito. In Peru the German psychologist Walter Blumenfeld, forced to leave Germany in the years of Hitlerian regime, worked since 1935 at the San Marcos University and was a pioneer of the objective orientation of psychology in this country.

Keywords

European psychologists; Andean countries; $20^{\text {th }}$ century 
Aunque los Andes se extienden a lo largo de toda la franja occidental de América del Sur, desde Venezuela hasta Chile, cuando se habla del mundo andino se hace referencia, por lo general, a tres naciones atravesadas por esa cordillera: Bolivia, Ecuador y Perú. Las tres surgieron a la vida independiente en la tercera década del siglo XIX, como resultado de la gesta liberadora que recorrió todo el subcontinente (excepto Brasil), y, desde entonces, a la geografía desafiante que las caracteriza, se ha unido también una historia accidentada, en la que revueltas, golpes de Estado y situaciones de marcada injusticia son denominadores comunes. Bolivia, Ecuador y Perú presentan indicadores socioeconómicos que se encuentran, aún hoy, entre los más preocupantes de Sudamérica (Portes \& Hoffman, 2003).

Perú y Bolivia, además, han protagonizado dos de los conflictos bélicos más sangrientos ocurridos en la región: en 1879 la Guerra del Pacífico (contra Chile) y, entre 1932 y 1935, la Guerra del Chaco (entre Bolivia y Paraguay). En Perú, el movimiento terrorista Sendero Luminoso desplegó su cruenta actividad entre 1980 y 1992. Problemas fronterizos entre Ecuador y Perú dieron lugar a un conflicto armado en 1942 y a escaramuzas en los años 1980 y 1990.

Estas tres naciones, como el resto de los países sudamericanos, se interesaron por atraer emigrantes europeos para poblar sus extensos territorios: "el fomento de la inmigración europea constituyó la obsesión de muchos gobiernos latinoamericanos" (Pellegrino, 1995, p. 183) ${ }^{1}$. Animadas sus elites políticas por contar con pobladores que pudieran emprender el proceso de desarrollo económico y social, que, según ellas, no podía ser llevado a cabo por la extendida población indígena (Martínez, 1997; Pellegrino, 1995), Ecuador, Bolivia y Perú buscaron la emigración europea.

Sin embargo, el éxito de este propósito fue más bien relativo. En tanto que Brasil, Argentina, Uruguay y Chile atrajeron un importante contingente de europeos (italianos, franceses, alemanes,

1 Dávila (2009) señala lo siguiente: "El proyecto político e ideológico de estas elites [las de América del Sur] se sintetizaba en fundar repúblicas independientes política y culturalmente, pero reteniendo los valores europeos y la supremacía blanca" (p. 358). holandeses, españoles, polacos) (Carducci, 2003; Domenech \& Magliano, s. f.; Martin \& Lovett, 1981), Bolivia, Ecuador y Perú recibieron un número muy limitado de emigrantes. No es el caso discutir las razones de esta emigración limitada. Queremos señalar solo, de un lado, la inestabilidad política de estas naciones y, del otro, el escaso nivel de desarrollo económico, así como las condiciones de vida particularmente difíciles ocasionadas por una geografía compleja, con ciudades a gran altura sobre el nivel del mar y con un clima caracterizado por extremos. Hay que agregar el hecho de la lejanía geográfica: Brasil, Argentina y Uruguay ofrecían rutas marítimas más cortas que, por ejemplo, el Perú. De otra parte, la vida cultural y científica en esas tres naciones andinas era muy limitada: un motivo más para que potenciales emigrantes europeos con intereses académicos optaran por países como Argentina o Brasil ${ }^{2}$.

En el presente trabajo queremos referirnos a un aspecto muy preciso de este tema de la emigración: la presencia de psicólogos europeos en esos tres países, en los que, como se sabe, la psicología existe como profesión desde hace muchos años (Alonso \& Eagly 1999). El término psicólogos será empleado en un sentido amplio: algunos de los estudiosos que pasaron una larga temporada en alguna o en varias de estas naciones en realidad no eran sensu stricto psicólogos, sino pedagogos o antropólogos, pero, como veremos, durante sus años en esos países llevaron a cabo investigaciones que tratan de temas cercanos a la psicología.

\section{Bolivia}

Bolivia ha despertado el interés de numerosos estudiosos, atraídos por su pasado, su diversidad étnica y la riqueza de recursos que se encuentra en ella. Cuando era el, así llamado, Alto Perú, el descubrimiento de las minas de plata en Potosí, en 1545, atrajo la atención de los europeos (Somarriva, 2007).

2 Si bien la Universidad de San Marcos, en Lima, era la más antigua de América del Sur, ella no podía competir con la Universidad de Buenos Aires, probablemente con una dotación económica mayor. 
En la primera mitad del siglo XIX, destaca de modo especial la figura de Alcide d'Orbigny, el gran naturalista francés que recorrió Bolivia entre 1830 y 1833 y que "fue el primero en describir -desde perspectiva amplia como 'abarcadora'-, la Bolivia profunda de los primeros años republicanos, estudiando en ella prácticamente la totalidad de sus múltiples y complejas facetas, muchas de ellas perdurables hasta hoy" (Arze Aguirre, 2003, p. 468; véase además el estudio detenido de Somarriva, 2007).

En el siglo XX, encontramos a Arthur Posnansky [Viena (Austria) 1873 - La Paz (Bolivia) 1946], que llevara a cabo una actividad sumamente versátil (minero, empresario, historiador, antropólogo y arqueólogo), desplegando además una producción escrita francamente sorprendente. Posnansky dedicó muchas de sus energías al estudio de la cultura Tiahuanaco, como lo demuestra el libro Tiahuanacu. La cuna del hombre americano (Posnansky, 1945, 1957).

Pero, por la misma época de Posnansky y sus planteamientos sobre la cultura Tiahuanaco, considerados por algunos como extremadamente especulativos (Lissner, 1957; Steward, 1946), encontramos a un pedagogo belga, Georges Rouma (1881-1976), contratado por el Gobierno boliviano de Ismael Montes en 1905, para crear un nuevo sistema educativo en ese país, junto con un grupo de profesores $^{3}$, conocido como "la misión belga", a fin de que "realizara un diagnóstico de la educación en la república y creara un nuevo sistema de enseñanza primaria, secundaria y normal "moderno" que encajara con los postulados liberales y europeizantes que gobernaban el país" (Yapu, 2011, p. 23)4.

3 Iño Daza (2012a) escribe sobre el contrato de Rouma lo siguiente: "En el momento en que el gobierno de Bolivia contrataba a Rouma él era un joven investigador especializado, de 25 años de edad. Rouma tenía una formación pedagógica en las nuevas formas de entender la educación moderna, por ello en sus planteamientos pedagógicos planteaba una educación que tome en cuenta los elementos psicológicos, físicos y sociales del entorno sociocultural en el cual vive el niño y el ser humano" (p. 426).

4 La misión llegó por partes entre 1911 y 1912 y estaba conformada por pedagogos y psicólogos: Adhémar Gehain (profesor de historia y geografía y bibliotecario), Emile Jacobs, Faría de Vasconcelos (1880-1939), Constant Lurquin, Henri De Genst, Julian Ficher, Henri Mettewie (enviado a Cochabamba, donde funda la Escuela
Nacido en Bruselas, Rouma se graduó como licenciado en ciencias antropológicas y profesor en pedagogía (Siñani Nina, 2007), colaborando alrededor de siete años con Oliver Decroly (18711932) (Iño Daza, 2012a), el famoso pedagogo belga, quien prologó su La parole et les troubles de la parole (Rouma, 1907), donde puede leerse que, cuando se encontraba en Europa, se desempeñaba como "professeur de pédagogie et de psychologie à l’École Normale Provinciale de Charleroi" y "directeur de la Section des Troubles de la Parole à la Policlinique de Bruxelles". El libro apareció en español en 1920 (Rouma, 1920).

Rouma fue nombrado director de educación primaria, secundaria y normal en Bolivia, e intentó desarrollar un sistema educativo apropiado a las características de la sociedad boliviana, más que copiar modelos europeos. En sus años en Bolivia, fundó varias escuelas normales rurales. En Sucre, tuvo a su cargo la Escuela Normal de Profesores y Preceptores de la República, fundada el 6 de junio de 1909 (Iño Daza 2012a; Lozada Pereira, 2009) y, finalmente en 1917, fundó el Instituto Normal Superior-Simón Bolívar en La Paz (Iño Daza, 2012b). Además, siempre según Siñani Nina, fundó en 1919 la Sociedad Nacional de Educación de Bolivia. Se dio tiempo, asimismo, Rouma para llevar a cabo estudios de naturaleza antropológica en 1911, cuyos resultados han quedado plasmados en publicaciones (p. ej., Rouma, 1913a), y de los que informó en

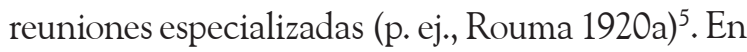
1913, fue Director General de Educación y en 1918 se trasladó a Cuba.

Ciertamente, los intereses antropológicos de Rouma persistirían a pesar del paso del tiempo y

\footnotetext{
de Artes y Oficios) y Raphael Segheers (Chávez Taborga, 2003; ver además, La Reforma Educacional en Bolivia de 1917).

5 Sobre el particular Martínez (1999) señala que "aprovechando un aura creciente de la antropología, el gobierno mandó a Rouma y a un equipo para que estudiaran a los indios aymaras y quechuas del Altiplano. Ellos eran básicamente lo que se consideraba como "la inmensa masa indígena” que el Censo de 1900 había destacado, pues las tribus del Oriente o del Chaco boliviano todavía eran vistas como tribus salvajes o, según la terminología del Censo, como "los no sometidos". El indio aymara o quechua era el que se debía conocer con más urgencia pues a él se pretendía incorporarlo cuanto antes a la nación, como fuerza productiva que
} participara del progreso nacional" (p. 376). 
de su alejamiento de Bolivia, país al que volvería en 1931 para una evaluación de los proyectos pedagógicos. A comienzos de la década de los 1920, publicaría El desarrollo físico del escolar cubano, sus curvas normales de crecimiento. Estudio de antropometría pedagógica (Rouma, 1920b). Para ese entonces, se presentaba en la obra antes mencionada como "Ex director general de instrucción pública en Bolivia" y como "asesor técnico de la Secretaría de Instrucción Pública y Bellas Artes”.

Con referencia a su trabajo pedagógico en Bolivia, existe información desperdigada en diarios y monografías, por lo general de difícil acceso. En 1916, sin embargo, Rouma dio a la publicidad un amplio informe (154 páginas) de sus actividades sobre el particular (Rouma, 1916). Otros trabajos suyos, asimismo difíciles de conseguir, ofrecen información sobre aspectos parciales de su trabajo en la nación andina (Rouma, 1912, 1913a, 1913b).

El trabajo de Rouma en Bolivia no dejó de despertar críticas. A pesar de que concedía un importante rol a los factores sociológicos en el proceso educativo (Rouma 1914, 1915) ${ }^{6}$, su visión, también influida por el darwinismo social, lo llevaba a considerar al niño boliviano como alguien con capacidades limitadas para el razonamiento y las inferencias, pero dotado de un sentido de la imaginación muy desarrollado. Una de las críticas más severas de sus ideas y de su labor provino de la pluma del educador español Joan Bardina i Castarà (1877-1950) (Bardina, 1917) , que desde 1917 se radicó en La Paz invitado por el Gobierno para dirigir la Normal Superior (Álvarez Gallego, 2011) y después emigró a Chile.

Debemos, por último, mencionar a Erhart Löhnberg, cuya difícil trayectoria en Bolivia merece ser destacada. Nacido en Alemania en 1903 y entrenado en el oficio de jardinero, Löhnberg lleva a cabo desde 1924 estudios universitarios en ciencias naturales, psicología y sociología en Erlangen,

6 Pérez-Serrano (2004) lo considera, junto con Paul Bergemann (1862-1946), George S. Counts (1889-1974), Fernando de Azevedo (1894-1974) y Émile Durkheim (1858-1917), como representante de la pedagogía sociológica.

7 Las críticas de Bardina a Rouma han sido expuestas en detalle en Martínez (2005)
Zurich, Bonn, Jena y Berlín, en donde obtendrá el doctorado en 1933, con un trabajo titulado "Die Rolle der Nachahmung bei den primitiven Völkern" (Löhnberg, 1933). Ya desde sus años de estudiante forma parte de grupos vinculados al partido comunista. Tras el doctorado se gana la vida como profesor a domicilio en familias judías. En 1937, se traslada a Suiza, después de haber sido sometido a interrogatorios por la Gestapo. Con posterioridad, lo encontramos en Bélgica, donde se entrenará como mecánico. En 1938, se traslada a Bolivia.

En ese país, Löhnberg se instala en Tarija donde se desempeñará como profesor de física en una escuela secundaria. Allí tuvo que enfrentarse a los ataques e intrigas de un grupo de alemanes seguidores de la doctrina nacionalsocialista que se empeñaron en desacreditarlo y en separarlo de la docencia. En 1944, opta (por razones de seguridad) por trasladarse a La Paz. En esa ciudad, permanecerá hasta 1951 desarrollando actividades periodísticas para Das andere Deutschland (DAD), una asociación de inmigrantes alemanes en Buenos Aires opuesta al régimen nacionalsocialista (Geuter \& León, 1990) ${ }^{8}$ y trabajando como profesor de idioma alemán. Es también secretario del grupo DAD en la capital boliviana y también secretario de la Sociedad de Inmigración y Socorro a los Inmigrantes. En 1952, se traslada a Inglaterra, donde será profesor de matemáticas, y en 1960 vuelve a Alemania, trabajando como profesor de idiomas y como escritor. En 1978, vivía en Berlín (Röder \& Strauss, 1980). No se sabe la fecha de su fallecimiento.

\section{Ecuador}

Ecuador también ha despertado permanente interés entre los científicos y estudiosos. Tal vez el más conocido de todos los que visitaron Ecuador sea Alexander von Humboldt (1769-1859), el gran investigador alemán, que en 1802 estudió el volcán

8 Das andere Deutschland existió entre 1937 y 1949, cuando fue disuelta debido a que, tras el derrumbe del nacionalsocialismo, su existencia carecía de sentido. Algunos de sus integrantes retornaron a Alemania (Friedman, 2010). Esta asociación daba a la luz una publicación con el mismo título, en la cual Löhnberg publicó varios trabajos entre 1942 y 1945 (Wittebur, 1991). 
Chimborazo, al que -erróneamente- consideró la montaña más alta del mundo (Meyer-Abich, 1967; Schaumann, 2009).

No debe olvidarse, asimismo, la visita que llevara a cabo nada menos que Darwin a bordo del Beagle, concentrada especialmente en las islas Galápagos?.

En materia de psicología y psiquiatría, el trabajo de Julio Endara (1899-1969) fue de decisiva importancia (Landázuri Camacho, 2008; Mariátegui, 1969). Psiquiatra de profesión y hombre de amplios horizontes culturales, Endara ha legado una importante obra escrita que trataba sobre temas de su especialidad, pero también sobre la psicología y la filosofía (ej., Endara, 1922, 1930, 1967).

La criminología fue también un área de interés de Endara quien, desde 1926 como profesor de psiquiatría en la Facultad de Medicina de la Universidad Central del Ecuador, fundó en 1936 el Instituto de Criminología en la Facultad de Jurisprudencia de la misma casa de estudios, y animó durante años los Archivos de criminología, neuro-psiquiatría y disciplinas conexas, publicado inicialmente en 1937, y cuya segunda época se inició en 1953, con amplia difusión internacional (Del Olmo, 1981).

La revista editada por Endara fue en su momento la más importante publicación periódica especializada del Ecuador, en el área de lo que hoy llamaríamos de manera amplia la salud mental. No debe sorprendernos por ello que en sus páginas aparecieran trabajos de especialistas de la psicología, la psiquiatría, la neurología y la criminología. Férenc Oliver Brachfeld (1908-1967), por ejemplo, dio a conocer en sus páginas, entre otros trabajos, una breve historia de la psicosíntesis (Brachfeld, 1953a, 1953b, 1955), una corriente que él trataba de difundir en esta parte del mundo desde un instituto que creara en Venezuela (Oberst, Ibarz, \& León, 2004).

Brachfeld, seguidor de las ideas de Alfred Adler (1870-1937) (Ibarz \& Villegas, 2002; León, 2000), era un psicólogo húngaro con sorprendentes habilidades lingüisticas que le permitieron dominar el alemán, el castellano y el catalán entre otros idio-

9 Darwin, por lo demás, también estuvo en El Callao, Perú, antes de trasladarse a las Islas Galápagos, en 1835. mas. Había emigrado a Venezuela después de residir durante años en España y Francia y, a mediados de la década de los 1960, se trasladó, por encargo del Ministerio de Cultura y Educación de la República Federal de Alemania (para el que trabajaba tras optar la nacionalidad alemana) primero a Colombia (1965-1966) y después a Ecuador (desde 1966), donde fallecería inesperadamente en 1967 (Ibarz \& Villegas, 2002; León, 1997, 2012).

Una de las razones por las cuales se trasladó a Ecuador fue, sin duda, el hecho que su instituto fuera clausurado en Venezuela (Camacho, 2008). Es de suponer que en sus frecuentes participaciones en congresos y otras actividades académicas trabara amistad con profesionales ecuatorianos. De hecho, Brachfeld tenía excelentes contactos no solo en América Latina, sino también en Europa. A su llegada a Ecuador, disponía de buenos amigos en ese país: aparte de Julio Endara, Emilio Uzcátegui García, educador, y Gustavo Alfredo Jácome, asimismo pedagogo; también a Edmundo Carbó, a quien es probable que conociera en el Primer Congreso Interamericano de Psicología, celebrado en Santo Domingo en 1953 (Gallegos, 2012a).

Anotemos, además, que Brachfeld era ya para entonces una figura bastante conocida en el ámbito de la psicología latinoamericana de la época: había sido presidente de la Sociedad Interamericana de Psicología entre 1953 y 1955 (Gallegos, 2012b).

Brachfeld pretendía llevar a cabo un amplio estudio sobre la psicología del hombre latinoamericano, y en el plano lectivo enseñó a los asistentes a sus cursos en la Universidad de Quito la terapia de "los sueños despiertos dirigidos", de Desoille (1890-1966) (Flachier Del Alcázar, s. f.). El estudio que proyectaba era muy probablemente una continuación de los planteamientos que había plasmado en Los sentimientos de inferioridad (Brachfeld, 1936), su obra más conocida y difundida, que, de otro lado, guardaba relación con las reflexiones llevadas a cabo por autores latinoamericanos acerca de la identidad latinoamericana (Arguedas, 1909/1979; Paz, 1992; Ramos, 1934; véase además, Gallo, 2010) y la sensación de minusvalía experimentada por muchos de los habitantes de esta parte del mundo. 


\section{Perú}

Perú es un país que ha atraído a numerosos científicos y estudiosos ${ }^{10}$. El caso paradigmático es el de Antonio Raimondi (1824-1890), botánico italiano radicado definitivamente en ese país, que contribuyó de modo sustantivo al conocimiento de la geografía, la historia y a la botánica peruanas.

En el Perú se registra la presencia a comienzos del siglo XX, el año 1903 para ser más preciso, de Isidore Poiry (1868-1954), pedagogo belga contratado por el Gobierno, como integrante de una misión de pedagogos ${ }^{11}$. Licenciado en ciencias naturales, Poiry publicó ese mismo año en francés un libro que lo presenta como "profesor en Bruselas en misión en Perú” (Poiry, 1903). En otra obra, aparecida años después, se lo presenta como "Exdirector fundador de la Escuela Normal de Lima" (Poiry, 1923; edición en francés, Poiry, 1919). Antes de fundar dicha escuela, Poiry fue en Lima subdirector del Colegio Guadalupe, donde enseñó alemán y química.

Influido por las ideas de la feminista y educadora sueca Ellen Key (1849-1926), que alcanzó celebridad mundial con su libro El siglo de los niños [originalmente en sueco: Barnets anhundrade, 2 vols., 1900] (Flores Quelopana, 2010) ${ }^{12}$, Poiry desarrolló en el Perú un programa de formación de educadores muy avanzado (Robles Ortiz, 2004), en el cual la psicología tenía un lugar importante. Ya en la Escuela Normal de Varones, tenía planeado instalar un laboratorio de psicología experimental (Elías, 1993).

Posteriormente, encontramos la presencia de tres emigrantes europeos: Maurice Simon (1900-?) y Hans Hahn (1900-1969), belga y alemán respectivamente, dejaron el Perú tras un tiempo, mientras que Walter Blumenfeld (1882-1967), alemán, permanecería hasta el fin de sus días en el Perú y

10 El ya mencionado Alexander von Humboldt también visitó el Perú, en donde tuvo oportunidad de encontrarse con Hipólito Unanue (1755-1833), importante figura de la ciencia y la cultura en los años postreros del Perú virreinal (Rizo Patrón, 2004; véase también Sobrevilla, 2002).

11 Los pedagogos contratados, todos ellos belgas, fueron Julio Becker, Julian Melckebecke, Leon Servais y Augusto Borms (Elias, 1993).

12 Sobre Key, su vida y la recepción de su obra en el mundo entero, véanse Lengborn (1993) y Ottavi (2005). desempeñaría un rol importantísimo en la constitución de la corriente objetivo-experimental de la psicología en ese país.

Sobre Maurice Simon se sabe relativamente poco, excepto que formó parte del Instituto Psicopedagógico Nacional, institución de alto nivel dedicada a la investigación pedagógica, psicológica y antropológica creada por el Gobierno peruano a comienzos de la década de los 40 (Alarcón, 2000; Ardila, 1986). Simon ha dejado testimonios de sus actividades en el país, como lo demuestran una memoria del año 1945 (Simon, 1946?) y un libro sobre metodología especial aplicada a la enseñanza primaria (Simon, 1940). Publicó, además, un tratado de psicología general (Simon, 1944), hoy olvidado.

Hans Hahn (1900-1969), psicotécnico alemán, tuvo un paso accidentado por el Perú, primero en Trujillo, en donde fundó un pequeño laboratorio de psicología experimental, y después en Lima, ciudad en la que permaneció un tiempo no muy largo, antes de emigrar a los Estados Unidos en situaciones poco claras (Orbegoso, 2011) ${ }^{13}$. Aunque su nombre está olvidado, Hahn, ya en Lima, participó en investigaciones sobre el efecto de la altura en los pobladores andinos. Sobre estos trabajos, y en particular en torno a su contribución a los mismos, reina el desconocimiento (León, 1993).

El nombre de Walter Blumenfeld es el de mayor importancia para la psicología peruana. Formado como ingeniero electricista y como psicólogo en Alemania, optó por el doctorado correspondiente con tesis dirigida por Carl Stumpf (1848-1936), un estudio clásico de la psicofísica de la visión (Blumenfeld 1913), desplegó una importante carrera académica en su país, trabajando como docente en la Universidad Técnica de Dresde, hasta que, con el ascenso de Hitler al poder y el partido nacionalsocialista, fue despojado de su cátedra y obligado a emigrar. La Universidad Nacional Mayor de San Marcos lo contrató para dirigir un instituto recién creado para el desarrollo de la psicotécnica y de las

13 Hace muchos años, Arnaldo Cano (1919-1987), psiquiatra peruano que desempeñó un rol importante en la formación universitaria de los primeros psicólogos en el país andino, señaló al autor de este trabajo, que corrió el rumor de que Hahn se había visto obligado a dejar el Perú debido a sospechas de espionaje. 
pruebas de admisión a esa casa de estudios (Alarcón, 1994, 2000).

Los primeros años de Blumenfeld en el Perú no estuvieron exentos de dificultades: su perspectiva de la psicología no tuvo una acogida favorable, pues predominaba en el mundo académico peruano un enfoque de naturaleza filosófica, representado por Psicología (Delgado \& Iberico, 1933), el libro de Honorio Delgado (1892-1969) y Mariano Iberico (1892-1974), quienes - de otro lado- parecen haber obstaculizado su carrera en el Perú y haber influido para que el instituto sanmarquino que dirigía Blumenfeld fuera cerrado a pocos años de su creación.

Sin embargo, Blumenfeld permanecería el resto de su vida en el Perú, siempre vinculado a la institución sanmarquina, y contribuyendo de modo decisivo al desarrollo de la vertiente científicoexperimental en el país andino. A comienzos de la década de los 1940, participó en calidad de directivo del ya mencionado Instituto Psicopedagógico Nacional (León, 1983). Autor de importantes libros como su Introducción a la psicología experimental (Blumenfeld, 1946) y su Psicología del aprendizaje (Blumenfeld, 1957), dos obras que alcanzaron varias ediciones, desplegó asimismo una activa labor como investigador (p. ej., Blumenfeld, 1943, 1949), y puede ser considerado como un precursor de lo que hoy se denomina psicología transcultural (Blumenfeld, 1952), al proponer una "vergleichende Ethnopsychologie" [etnopsicología comparada].

Cumplió también una importante tarea formativa de los cuadros que, con el tiempo, asumirían el liderazgo de la psicología como ciencia y como profesión en el Perú. Destaca entre sus más distinguidos discípulos, Reynaldo Alarcón, autor de una importante obra escrita que abarca la historia de la psicología, la psicometría y, recientemente, la psicología positiva (Alarcón, 2012).

Con el paso del tiempo, Blumenfeld, que en los años 50 trabajó activamente en el campo de la pedagogía e hizo incursiones en el terreno de las tipologías psicológicas (Blumenfeld, 1958), se inclinó cada vez más hacia los temas filosóficos, proponiendo a la gratitud como fundamento de la ética (Blumenfeld, 1961, 1966).

\section{Comentario final}

Es interesante anotar el interés que estos países, al otro lado de los Andes desde la posición de Europa, despertaron entre científicos y estudiosos. Hemos señalado el caso de Raimondi para el Perú, Posnansky para Bolivia, y el más conocido, de Alexander von Humboldt para Ecuador. El exotismo, la presencia de zonas absolutamente vírgenes, la riqueza cultural y la diversidad biológica fueron poderosos atractivos para muchos espíritus europeos, como aún hoy lo continúan siendo.

Pero en el caso de la psicología, el interés no fue tan grande: el número de psicólogos que se radicó en estos países fue muy reducido. En Chile y Colombia, dos países igualmente recorridos por la cordillera de los Andes, la situación tampoco fue muy diferente. En Chile, Béla Székely (1892-1955), un psicólogo húngaro también cercano a la corriente adleriana, estuvo por algún tiempo trabajando en la Universidad Católica (en 1954), en Santiago (Bravo, 2004; Hopfengärtner, 2012), después de haber estado largo tiempo en Buenos Aires. En Colombia, Mercedes Rodrigo (1901-1982), emigrante española, trabajó en la Universidad Nacional, en Bogotá, pero se vio obligada a trasladarse a Puerto Rico por razones políticas (Ardila, 2013).

Es probable que algunos más pensaran en radicarse en Bolivia, Ecuador y Perú, pero una serie de factores o el encontrar mejores oportunidades, hizo que al final se establecieran en otros países. Tal parece haber sido el caso de Emilio Mira y López (1896-1964), reconocida figura de la psicotécnica en el siglo XX, quien, obligado a dejar España, se trasladó a Argentina y, desde allí, en una carta a Walter Blumenfeld le comunica que existió alguna posibilidad de emigrar al Perú y enseñar en la Universidad de San Marcos, pero que Honorio Delgado, psiquiatra peruano e influyente intelectual, se había opuesto (León \& Kagelmann, 1991).

Tanto en Bolivia como en Perú muchos de los psicólogos y pedagogos europeos que estuvieron en esas naciones lo hicieron en el marco de misiones pedagógicas, cuya finalidad era reorganizar el sistema de enseñanza primaria y secundaria. Por cierto, no fueron los únicos países que recurrieron 
a tales misiones. También en Chile se contrató a una serie de pedagogos, estos más bien alemanes, para mejorar la educación en ese país (Bravo, 2004; Salas \& Lizama, 2011). La presencia de misiones pedagógicas europeas en estos países fue la expresión del deseo de modernizar los sistemas educativos locales. Europa ofrecía modelos de educación (Froebel, Decroly, Montessori) que fueron vistos con interés por los gobiernos latinoamericanos.

Bruselas, en particular, fue en los años previos a la Primera Guerra Mundial uno de los centros más activos a nivel mundial en la investigación infantil (Depaepe, 1993). Decroly, ya en 1901, establecía el proyecto de una escuela para niños especiales (École d'Enseignement Spécial pour Enfants Irreguliers), que alcanzó gran difusión en el mundo entero y también en América del Sur (Caiceo, 2011; Dubreucq, 1992). Él y Montessori visitarían América Latina: Decroly estuvo en Colombia en 1925 (Torres Cruz \& Londoño Ramos, 2011) y Montessori, en Buenos Aires.

La información sobre el tema que hemos presentado es fragmentaria y de difícil acceso (dado que está muy desperdigada), especialmente en el caso de Bolivia y Ecuador. Hace falta llevar a cabo más investigación histórica, a fin de reconstruir periodos y redescubrir protagonistas, hoy día tal vez olvidados o absolutamente ignorados.

\section{Referencias}

Alarcón, R. (1994). El pensamiento psicológico de Walter Blumenfeld. Lima: Concytec.

Alarcón, R. (2000). Historia de la psicología en el Perú. De la Colonia a la República. Lima: Universidad Ricardo Palma.

Alarcón, R. (2012). Psicología y testimonio personal. En H. Klappenbach \& R. León (Eds.), Historia de la psicología iberoamericana en autobiografías (pp. 21-66). Lima: Universidad Ricardo Palma/ Sociedad Interamericana de Psicología.

Alonso, M. M., \& Eagly, A. H. (Eds.). (1999). Psicología en las Américas. Caracas: Sociedad Interamericana de Psicología.
Álvarez Gallego, A. (2011). Miguel Fornaguera, un librepensador catalán en Colombia. Revista Colombiana de Educación, 61, 299-316.

Ardila, R. (1986). La psicología en América Latina. Pasado, presente y futuro. México: Siglo XXI.

Ardila, R. (2013). Historia de la psicología en Colombia. México: Manual Moderno.

Arguedas, A. (1979). Pueblo enfermo. La Paz: Gisbert. (Trabajo original publicado en 1909)

Arze Aguirre, R. D. (2003). Alcide d'Orbigny en la visión de los bolivianos. Bulletin de l'Institut Français d'Études Andines, 32(3), 467-477.

Bardina, J. (1917). Arcaísmos de la misión belga. La Paz: Tipografía América.

Blumenfeld, W. (1913). Untersuchungen über die scheinbare Grösse im Sehraume. [Investigaciones sobre el tamaño aparente en el espacio visual] Zeitschrift für Psychologie, 65, 241-404.

Blumenfeld, W. (1943). Los rendimientos en el test colectivo de Terman en su dependencia de la edad cronológica y el grado escolar. Boletín del Instituto Psicopedagógico Nacional, 2, 3-28.

Blumenfeld, W. (1949). Análisis de las tendencias a la introversión y la extraversión en el Perú y en los Estados Unidos a base del inventario de la personalidad de Bernreuter. Boletín del Instituto Psicopedagógico Nacional, 8, 3-33.

Blumenfeld, W. (1946). Introducción a la psicología experimental. Lima: Cultura Antártica.

Blumenfeld, W. (1952). Erfahrungen mit Intelligenz- und charakterologischen Tests in Peru und ihre Beziehungen zum Problem einer vergleichenden Ethnopsychologie. [Experiencias con tests de inteligencia y caracterológicos en el Perú y sus relaciones con el problema de una etnopsicología comparada] En F. Baumgarten (Ed.), Lapsychotechnique dans le monde moderne (pp. 517-527). París: PUF.

Blumenfeld, W. (1957). Psicología del aprendizaje. Lima: Universidad Nacional Mayor de San Marcos.

Blumenfeld, W. (1958). Don Quijote und Sancho Panza als psychologische Typen. [Don Quijote y Sancho Panza como tipos psicológicos] Psychologische Rundschau, 9, 29-52.

Blumenfeld, W. (1961). Los fundamentos de la ética y el principio generalizado de la gratitud. Lima: Universidad Nacional Mayor de San Marcos. 


\section{Psicólogos europeos en los países andinos (Bolivia, ECUAdor}

Y PERÚ) DURANTE LA PRIMERA MITAD DEL SIGLO XX

Blumenfeld, W. (1966). Contribuciones críticas y constructivas a la problemática de la ética. Lima: Universidad Nacional Mayor de San Marcos.

Bolivia, Ministerio de Educación. (1917). La reforma educacional en Bolivia. La Paz: Autor.

Brachfeld, F. O. (1936). Los sentimientos de inferioridad. Barcelona: Apolo.

Brachfeld, F. O. (1953a). Breve historia de la psicosíntesis. Archivos de Criminología, Neuro-Psiquiatría y Disciplinas Conexas, 1, 299-307.

Brachfeld, F. O. (1953b). Un criminólogo olvidado: Andreas Bjerre. Archivos de Criminología, NeuroPsiquiatría y Disciplinas Conexas, 1, 135-142.

Brachfeld, F. O (1955). El "fatoanálisis" de Szondi y la criminología. Archivos de Criminología, NeuroPsiquiatría y Disciplinas Conexas, 3, 457-467.

Bravo, L. (2004). Cincuenta años de psicología en la Universidad Católica. Psykhe, 13(1), 197-204.

Caiceo, J. (2011). Desarrollo de la educación parvularia en Chile. Historia da Educaçao, 15(34), 22-44.

Camacho, C. (2008). Microhistoria de un fracaso: el Instituto de Psicosíntesis y Relaciones Humanas de la Universidad de los Andes (Venezuela) 1952. 1954. Procesos Históricos, 7(13), 213-249.

Carducci, L. G. C. (2003). L'emigrazione italiana in Bolivia dall'unità alla finne del XX secolo: periodizzacione e caractteristiche. [La emigración italiana en Bolivia de la "unidad" hasta fines del siglo XX: periodización y características] Altreitalia, julio-diciembre, 53-76.

Chávez Taborga, C. (2003). Guido Villa-Gómez en tres perfiles. (Estimativa Literaria). Sucre: Universidad Andina Simón Bolívar.

Del Olmo, R. (1981). América Latina y su criminología. México: Siglo XXI.

Delgado, H., \& Iberico, M. (1933). Psicología. Lima: Autores.

Depaepe, M. (1993). Zum Wohl des Kindes? Pädologie, pädagogische Psychologie und experimentelle Pädagogik in Europa und den USA, 1890-1940. [¿Por el bienestar del niño? Paidología, psicología pedagógica y pedagogía experimental en Europa y en los Estados Unidos, 1890-1940] Weinheim: Deutscher Studien Verlag.

Domenech, E. E., \& Magliano, M. J. (s. f.). Políticas migratorias en Bolivia: el estado nacional frente a las migraciones internacionales [Versión resumida del artículo "Migraciones internacionales y política en Bolivia: pasado y presente" (2007)]. Recuperado de http://webiigg.sociales.uba.ar/pobmigra/archivos/ DomenechMagliano\%20Aepa07.pdf

Dubreucq, F. (1992). Perfiles de educadores: Jean Ovide Decroly. Perspectivas, 22(3), 409-429.

Elias, H. (1993). La misión belga de 1903: una reforma de la educación peruana de permanente vigencia. Lima: Universidad de Lima.

Endara, J. (1922). José Ingenieros y el porvenir de la filosofía. Buenos Aires: Agencia General de Librería y Publicaciones.

Endara, J. (Comp.). (1930). Los temperamentos. Quito: Imprenta de la Universidad Central.

Endara, J. (1967). Test de Rorschach. Técnica, evolución y estado actual (2.a. ed.). Barcelona: CientíficoMédica.

Flachier Del Alcázar, J. (s. f.). Por los caminos de Alfred Adler. Recuperado de http://www.centroadleriano. org/publicaciones/Porloscaminos.pdf

Flores Quelopana, G. (2010). ¿Existe un proyecto formativo en el Perú? Umbral. Revista de Educación, Cultura y Sociedad, 9(19-20), 9-21.

Friedman, G. (2010). Alemanes antinazis en la Argentina. Buenos Aires: Siglo XXI.

Gallegos, M. (2012a). El Primer Congreso Interamericano de Psicología (1953): su acontecer histórico. Revista Interamericana de Psicología, 46(1), 21-34.

Gallegos, M. (2012b). Historia de la psicología interamericana: Sociedad Interamericana de Psicología (1951). Psychologia Latina, 3(1), 23-36.

Gallo, R. (2010). Freud's Mexico. Into the wilds of psychoanalysis. Cambridge/ Londres: The MIT Press.

Geuter, U., \& León, R. (1990). Flucht nach Südamerika - Europäische Emigranten in der lateinamerikanischen Psychologie. [Huída hacia América del Sur. Emigrantes europeos en la psicología latinoamericana] Psychologie und Geschichte, 1, 24-37.

Hopfengärtner, J. (2012). Apuntes para una biografía de Béla Székely (1892-1955). Revista de Psicología, 12,187-210.

Ibarz, V., \& Villegas, M. (2002). Ferenc Olivér Brachfeld (1908-1967). Revista de Historia de la Psicología, 23(3-4), 265-275. 
Iño Daza, W. G. (abril, 2012a). A más de cien años de la fundación de la "Escuela Normal de Profesores y Preceptores de la República” en Sucre (1909). Anales de la Reunión Anual de Etnología, N.o 24. Disponible en http://200.87.119.77:8180/musef/ handle/123456789/513

Iño Daza, W. G. (2012b). La reforma educativa liberal (1899-1920): modernización de la educación pública en Bolivia [Versión electrónica]. Estudios Bolivianos, 16.

Key, E. (1906). El siglo de los niños (Vols. 1-2). Barcelona: Imprenta de Henrich.

Landázuri Camacho, M. (2008). Salir del encierro. Medio siglo del Hospital Psiquiátrico San Lázaro. Quito: Banco Central del Ecuador.

Lengborg, Th. (1993). Ellen Key (1849-1926). Perspectivas. Revista Trimestral De Educación Comparada, 23(3-4), 873-886.

León, R. (1983). Un pionero de la psicología en América Latina: Walter Blumenfeld. Revista Latinoamericana de Psicología, 15, 433-452.

León, R. (1993). Contribuciones a la historia de la psicología en el Perú. Lima: CONCYTEC.

León, R. (1997). Rumbo al nuevo mundo: cuatro psicólogos de Europa Oriental en la historia de la psicología de América del Sur. Revista Latinoamericana de Psicología, 29(1), 9-34.

León, R. (2000). Los psicólogos hispanohablantes y la teoría de Alfred Adler en la revista Internationale Zeitschrift für Individualpsychologie (1914-1937). Revista Latinoamericana de Psicología, 32(1), 107126.

León, R. (2012). F. Oliver Brachfeld y Werner Wolff: dos figuras en los inicios de la Sociedad Interamericana de Psicología. Revista Interamericana de Psicología, 46(1), 35-42.

León, R., \& Kagelmann, H. J. (1991). Zwei Emigranten in Südamerika: Der Briefwechsel zwischen Walter Blumenfeld und Emilio Mira y López. [Dos emigrantes en América del Sur: la correspondencia entre Walter Blumenfeld y Emilio Mira y López] Psychologie und Geschichte, 3, 65-76.

Lissner, I. (1957). Nuestro antepasado el hombre. 7000 años de civilización. Barcelona: Jano.
Löhnberg, E. (1933). Die Typen der Nachahmung bei den primitive Völkern (Tesis doctoral). FriedrichWilhelms-Universität, Berlín.

Losada Pereira, B. (2009). La historia de la formación docente en Bolivia comparada con las tendencias educativas de Latinoamérica y el Caribe. Estudios Bolivianos, 15, 7-56.

Mariátegui, J. (1969). In memoriam Julio Endara (1899. 1969). Revista de Neuro-Psiquiatría, 32, 237-238.

Martín, M. R. H., \& Lovett, G. H. (1981). Encyclopedia of Latin American History. Westport, CT: Greenwood Press.

Martínez, F. (1997). Apogeo y decadencia del ideal de la inmigración europea en Colombia, siglo XIX. Boletín Cultural y Bibliográfico, 34(44), 3-45.

Martínez, F. (1999). iQué nuestros indios se conviertan en pequeños suecos! La introducción de la gimnasia en las escuelas bolivianas. Bulletin de l'Institut Français d'Études Andines, 28(3), 361-386.

Martínez, F. (2005). Les tribulations de Juan Bardina en Espagne, en Bolivie et au Chili, et les diffèrentes récupérations nationales de sa mémoire. En J. Farré (Ed.), Hommes de science et intellectuels européens en Amérique latine (XIXe-XXe siècles) (pp. 339-358). París: Éditions Le Manuscrit.

Meyer-Abich, A. (1967). Alexander von Humboldt. Hamburgo: Rowohlt.

Oberst, U., Ibarz, V., \& León, R. (2004). La psicología individual de Alfred Adler y la psicosíntesis de Olivér Brachfeld. Revista de Neuro-Psiquiatría, 67, 31-44.

Orbegoso, A. (2011). Hans Hahn, iniciador de la psicología experimental en Trujillo (Perú). Revista de Psicología, 13(2), 235-240.

Ottavi, D. (2005). Ellen Key et le "puérocentrisme". En J. Carroy, N. Edelman, A. Ohayon \& N. Richard (Dirs.), Les femmes dans les sciences de l'homme (XIXe-XXe siècles) (pp. 31-48). París: Seli Arslan.

$\mathrm{Paz}$ O. (1992). El laberinto de la soledad. México: Fondo de Cultura Económica. (Trabajo original publicado en 1950)

Pellegrino, A. (1995). La migración internacional en América Latina. Pensamiento Iberoamericano, 28, 177-210.

Pérez Serrano, G. (2004). Pedagogía social - Educación social: construcción científica e intervención práctica. Madrid: Narcea. 


\section{Psicólogos europeos en los países andinos (Bolivia, ECUAdor}

Y PERÚ) DURANTE LA PRIMERA MITAD DEL SIGLO XX

Poiry, I. (1903). Méthode directe et intuitive de la langue allemande por les écoles. Bruselas: J. Lèbègue.

Poiry, I. (1919). La réforme de l'education. Bruselas: J. Lèbègue.

Poiry, I. (1923). La reforma de la educación (A. García Martín, Trad.). Granada: Tip de El Defensor.

Portes, A. \& Hoffman, K. (2003). Latin American class structures: their composition and change during the neoliberal era. Latin American Research Review, 38, 41-82.

Posnansky, A. (1945). Tiahuanacu: The cradle of American man (Vols. 1-2). New York: J. J. Austin Publisher.

Posnansky, A. (1957). Tiahuanacu: The cradle of american man (Vols. 3-4). La Paz: Ministerio de Educación.

Ramos, S. (1934). El perfil del hombre y la cultura en México. México: Mundial.

Rizo Patrón, P. (2004). Arrogance and squalor? Lima's elite. En R. Erickson, M. Font, \& B. Schwartz (Coords.), Alexander von Humboldt. From the Americas to the cosmos (pp. 69-81). New York: Bildner Center for Western Hemisphere Studies.

Robles Ortiz, E. (2004). Las primeras escuelas normales en el Perú. Revista de Historia de la Educación Latinoamericana, 6, 57-86.

Röder, W., \& Strauss, H. A. (Eds.). (1980). Biographisches Handbuch der deutschsprachigen Emigration nach 1933. Vol. 1: Politik, Wirtschaft, Öffentliches Leben. Munich/New York/Londres/París: K. G. Saur.

Rouma, G. (1907). La parole et les troubles de la parole. París: Paulin.

Rouma, G. (1912). De Buenos Ayres à Sucre. En La vie intellectuelle, 4, 11-220.

Rouma, G. (1913a). Les indiens quitchouas et aymaras des hauts plateaux de la Bolivie: résultats de la Mission anthropologique organisée en 1911 sous les auspices du gouvernement de la République de Bolivie et de la Société anthropologique "Sucre" sous la direction de l'auteur. Bruselas: Misch \& Thron.

Rouma, G. (1913b). L'école normale de Sucre. École Moderne, 5-6, 233-257.

Rouma, G. (1914). Pédagogie sociologique. Les influences des milieux en éducation. Neuchâtel: Delachaux et Niestlé.

Rouma, G. (1915). Pedagogía sociológica. Los influjos del medio en la educación. Madrid: F. Beltrán.
Rouma, G. (1916). Informe del doctor Georges Rouma, director general de instrucción primaria, secundaria y normal, presentado a la consideración del señor ministro de educación pública. 1915-1916. La Paz: Imprenta Velarde.

Rouma, G. (8 de enero de 1920a). Le développement physique de l'écolier bolivien [Extracto]. París: Bulletins et Mémories de la Société d'Anthropologie de Paris.

Rouma, G. (1920b). El desarrollo físico del escolar cubano, sus curvas normales de crecimiento. Estudio de antropometría pedagógica. La Habana: Morlón.

Rouma, G., \& Arze, J. A. (1952). Sociografía del inkario: ifue socialista o comunista el imperio inkaiko? La Paz: Fénix.

Rouma, G., \& Decroly, O. (1920). La palabra y las perturbaciones de la palabra (D. Gonzalo \& R. Lafora, Trads.). Madrid: Francisco Beltrán.

Salas, G., \& Lizama, E. (2009). Historia de la psicología en Chile: 1889-1981. La Serena: Universidad de La Serena.

Schaumann, C. (2009). Who measures the world? Alexander von Humboldt's Chimborazo climb in the literary imagination. The German Quarterly, 8(4), 447-468.

Simon, M. (1940). Metodología especial: enseñanza primaria. Lima: Editorial Libr. Peruana.

Simon, M. (1944). Tratado de psicología (Vols. 1-2). Lima: D. Miranda.

Simon, M. (1946?). Instituto Psicopedagógico Nacional de Varones. Clausura del año académico de 1945. Memoria leída por el director Dr. Maurice Simon. Lima: Librería e Imprenta D. Miranda.

Siñani Nina, A. (7 de noviembre de 2007). Georges Rouma: un maestro "belga-boliviano". El Diario, p. 10.

Sobrevilla, D. (2002). La visión crítica de Humboldt de la sociedad peruana. Acta Herediana, 32, 17-34.

Somarriva, M. (2007). D’Orbigny y otros viajeros por el Alto Perú de comienzos del siglo XIX. En E. Cavieres (Ed.), Del altiplano al desierto. Construcción de espacios y gestación de un conflicto. Bolivia, Chile y Perú en el siglo XIX (pp. 209-244). Valparaíso: Ediciones Universitarias de Valparaíso.

Steward, J. H. (1946). Tiahuanacu: The cradle of American man [Book Reviews]. Science, 104(2688), 19 20. doi: 0.1126/science.104.2688.19 
Torres Cruz, D. L., \& Londoño Ramos, C. A. (2011). Textos y pedagogía en los albores del siglo XX en Colombia. Revista de Historia de la Educación Latinoamericana, 16, 255-278.

Wittebur, K. (1991). Die deutsche Soziologie im Exil, 1933. 1945: eine biographische Kartographie. MünsterHamburgo: Lit.
Yapu, M. (2011). Políticas educativas, interculturalidad y discriminación. Estudios de caso: Potosí, La Paz y El Alto. La Paz: Programa de Investigación Estratégica en Bolivia. 